\title{
An Efficient Image De-Blurring Technique Using Point Spread Function in High Definition Medical Image
}

\author{
Mohammed Anwar ${ }^{1} \&$ Abubakar Muhammad Ashir ${ }^{2}$
}

\author{
${ }^{1 \& 2}$ Department of Computer Engineering, Faculty of Engineering, Tishk International University, \\ Erbil, Iraq \\ Correspondence: Mohammed Anwar, Tishk International University, Erbil, Iraq \\ Email: muhammed.anwar@tiu.edu.iq
}

Doi: 10.23918/eajse.v6i1p27

\begin{abstract}
Medical image-enhancing technology plays a significant role for processing and revealing discerning information from acquired images in many applications such as Magnetic Resonance Imaging (MRI) and Computed Tomography (CT) which are frequently used for diagnosis and treatments in medical imaging. The clarity of these images become of great importance considering the details required to render diagnosis. The effects associated with blurred images in such applications is very challenging. The blurring effect is largely unavoidable due to the errors associated with capturing devices and natural motion in the human body. In this research, a method is proposed utilizing image blending approach to significantly reduce the effects of blur from an image through motion adaptive Point Spread Function (PSF). The proposed Efficient Image De-Blurring methods (EIDB) is realized using PSFs. And then get deblurred quality images from image de-blending image set in the alpha plane.
\end{abstract}

Keywords: Point Spread Function, Alpha Plane, Image Blending, De-Blurring, Deblurred Medical Images

\section{Introduction}

The health care imaging has an unfathomable occupation in suitable assurance and examination of a physiological piece of the human body. The helpful authorities energetically safeguard and depend on the imaging results procured from the image sensors (Fowler, AlGamal, \& Yang, 1994). Sadly, there is unavoidable development in the human body, for instance, a moving undeveloped organism in the paunch or a more seasoned person who can't adjust his position. Indeed, even in some cases, the picture catching a sensor or gadget might not have a sharp introduction time bringing about the obscured pictures. The obscured restorative pictures are hard to break down the physiology and arrive at a decent symptomatic outcome.

There have been numerous endeavors in the picture handling strategies like Removing Complication calculations, for example, and (EIDB) Efficient Image De-Blurring (Jiunn-Lin, Chang, \& Chen, 2013), Wiener Removing Complication (Lil, Meunier, \& Soucy, 2005) and Blind Removing Complication (Mane \& Pawar, 2014) to give some examples. Numerous scientists have proposed the (EIDB) Efficient Image De-Blurring methods with point spread capacity (PSF) to be the nearest estimate similarly as movement deblurring (Li \& Zhan, 2012) is considered. Yet at the same time it is a guess and there is the extent of progress (Sharma, 2016). A nearby investigate the complicated pictures got from directly shifted PSF work (Dong \& Xie, 2016) insights toward the adjustment in the edges of the reestablished pictures in an irregular manner.

The aim of this work is to provide a new approach which mitigates the effects of image de-blurring resulting from image capturing device error and image target motion. These factors led to an obscured image with insufficient clarity to reveal useful information. The proposed approach utilizes a mixing

Volume 6, Issue 1; June, 2020 
of pictures in the alpha plane to consolidate the details in the edges of complicated pictures by smoothing them out to give a crisper and more honed picture. An overview of varied point spread function is used to deblur and remove complications are depicted in Figure 1.

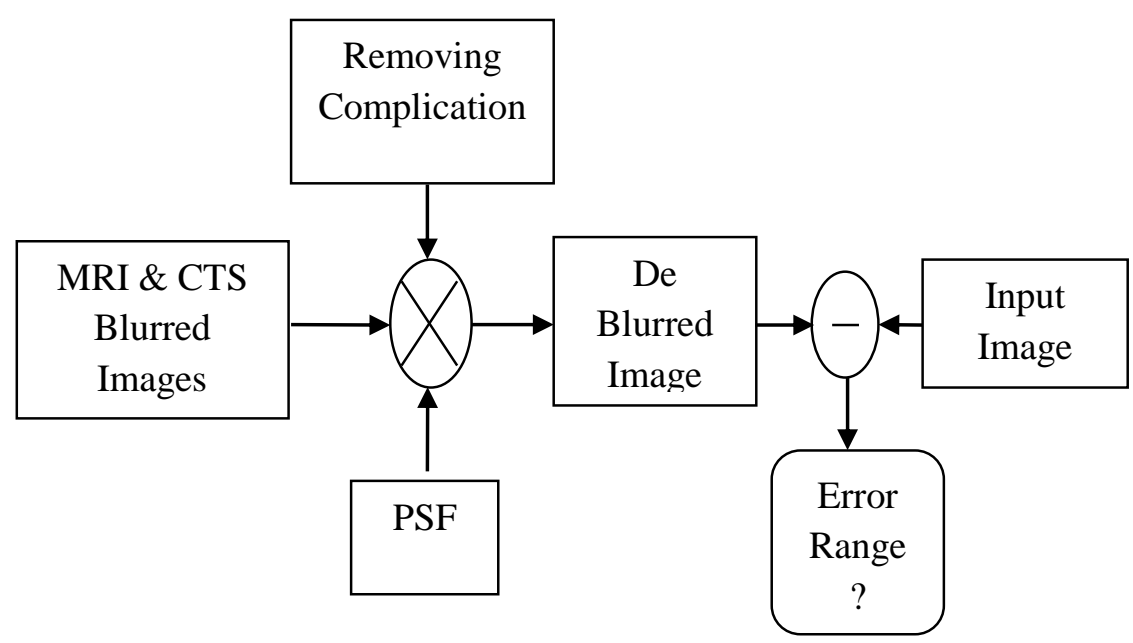

Figure 1: Varied PSF Removing Complication

The proposed method solves two major issues with blurred images. Firstly, it used PSF to reduce device-induced blur and then EIDB calculation were applied to achieve removing complication and target motion related blur. Along these lines, with changed PSF (Angelis \& Kyme, 2016) a lot of obscure pictures are acquired to be worked with alpha plane mixing. Five stages are involved in the proposed method. The first stage is the filtration of the Computed Tomography (CT) and Magnetic Resonance Imaging (MRI) obscured pictures as the dataset. The dataset is taken separately https://medpix.nlm.nih.gov/. In the second stage Removing Complication and PSF techniques were used with obscured pictures to deblur process. The third stage computes the differences or error between the deblurred and obscured pictures.

\section{Proposed Work}

The medicinal pictures are taken by MRI and CT utilizing different X-beams to get a cross-sectional perspective on the body and perspectives for life systems. In those cases, they may have moved obscured impacts. (EIDB) Efficient Image De-Blurring acquired absolutely five obscured pictures that are three from of MRI filter pictures and two from CT examine pictures. The obscured test picture is convolved with factor to get a similar arrangement of pictures in the obscured structure to make an example set with EIDB. Essentially, the pictures are irregular, yet they are picked a premise of grayscale (Yim, Choyke, \& Summers, 2000) variety to coordinate the authenticity in down to earth restorative situation (Pizurica \& Philips, 2003). Figures 2 and 3 indicate tests of obscured input pictures separately. 

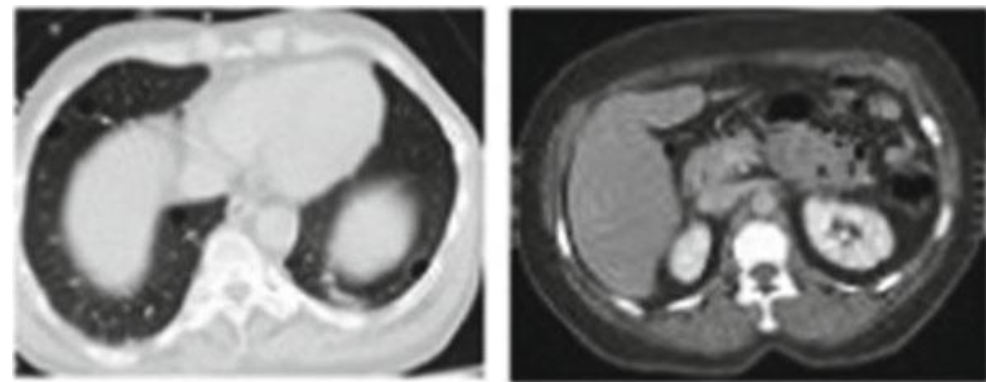

Figure 2: CT-1 and CT-2 processed tomography images
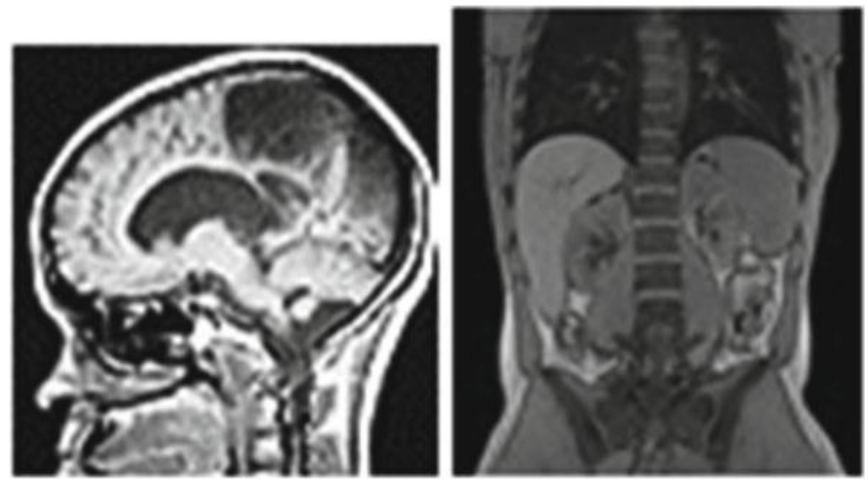

Figure 3: MRI-1 and MRI-2 magnetic resonance images

\subsection{Convolution Result for Blurring Image}

The example picture is obscured utilizing differed PSF with the assistance of roundabout convolution and commotion is likewise acquainted with model a constant estimate of obscured pictures as appeared in Figures 4 and 5.

Procedure: Blurring Sample Images

Attributes: Len $=21$; Theta $=11 ;$ CIR - Circular SI - Sample Image. NM - Noise Mean. NV - Noise Variable. BR - Blurred Image IMN - imnoise

Step 1: I2D (imread(SI));

Step 2: PSF motion with Len and Theta.

Step 3: imfilter (S, PSF, 'CV', 'CIR');

Step 4: NM 0;

Step 5: NV 0.0001 .

Step 6: BR + noisy IMN (blurred1, 'gaussian', NM, NV);

End: Blurring Sample Images 

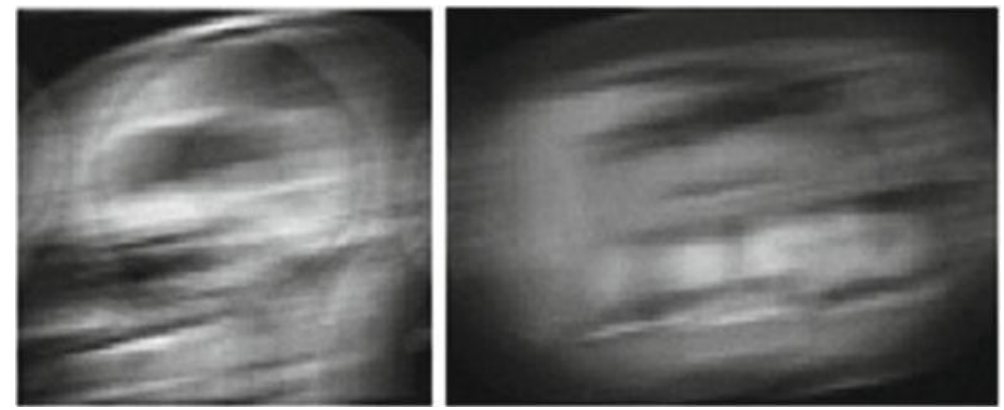

Figure 4: MRI-1 and CT-1 Blurred images
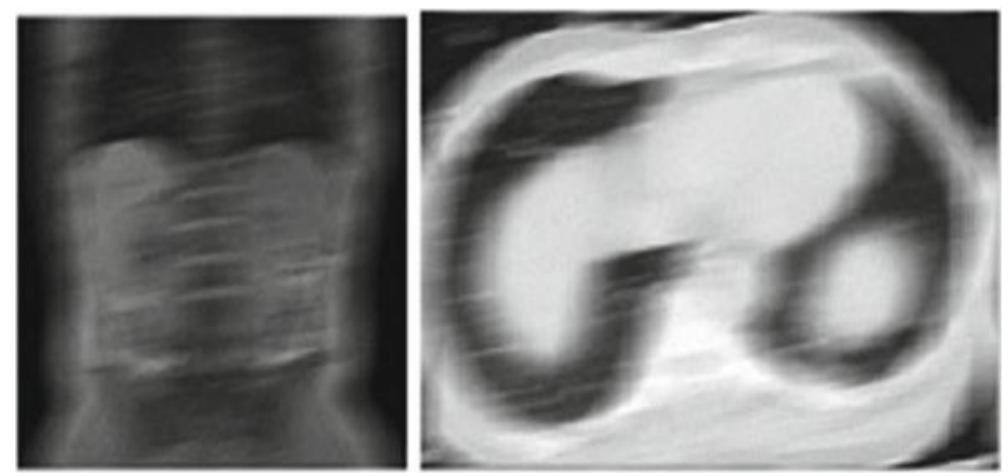

Figure 5: MRI-2 and CT-2 Blurred images

\subsection{D-L RC Method along PSFs Linearly Varying}

The obscured pictures are getting tests to deconvolve with differed PSF for deblurring. PSF_one, PSF_two, PSF_three, PSF_four, PSF_five and PSF_six have taken length 12, 13,14,15,16 and 17 separately.
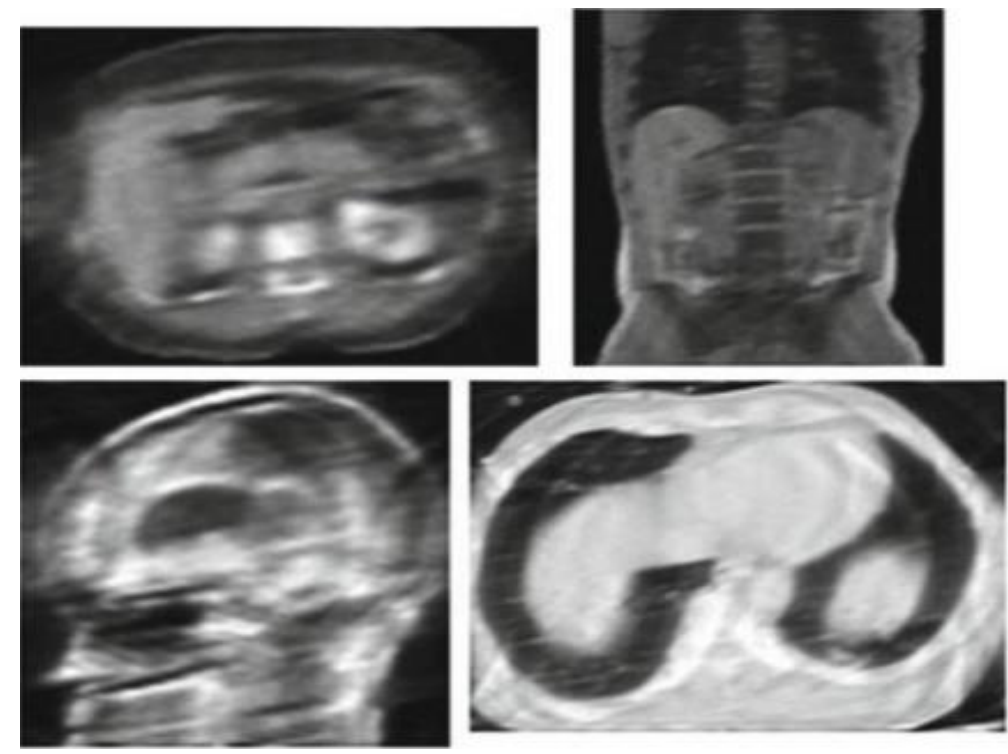

Figure 6: LRC images with PSF_one 
The consequence of pictures gets from PSF1. These appear in Figure 6. The Figure 7 shows the aftereffect of EIDB complicated with the length of PSF2 is 20. The Figures 6 and 7 are CT and MRI input pictures. Figures 8, 9, 10, and 11 indicated consequence of EIDB complicated pictures with PSF2 to PSF5 and where length 13 to 17.
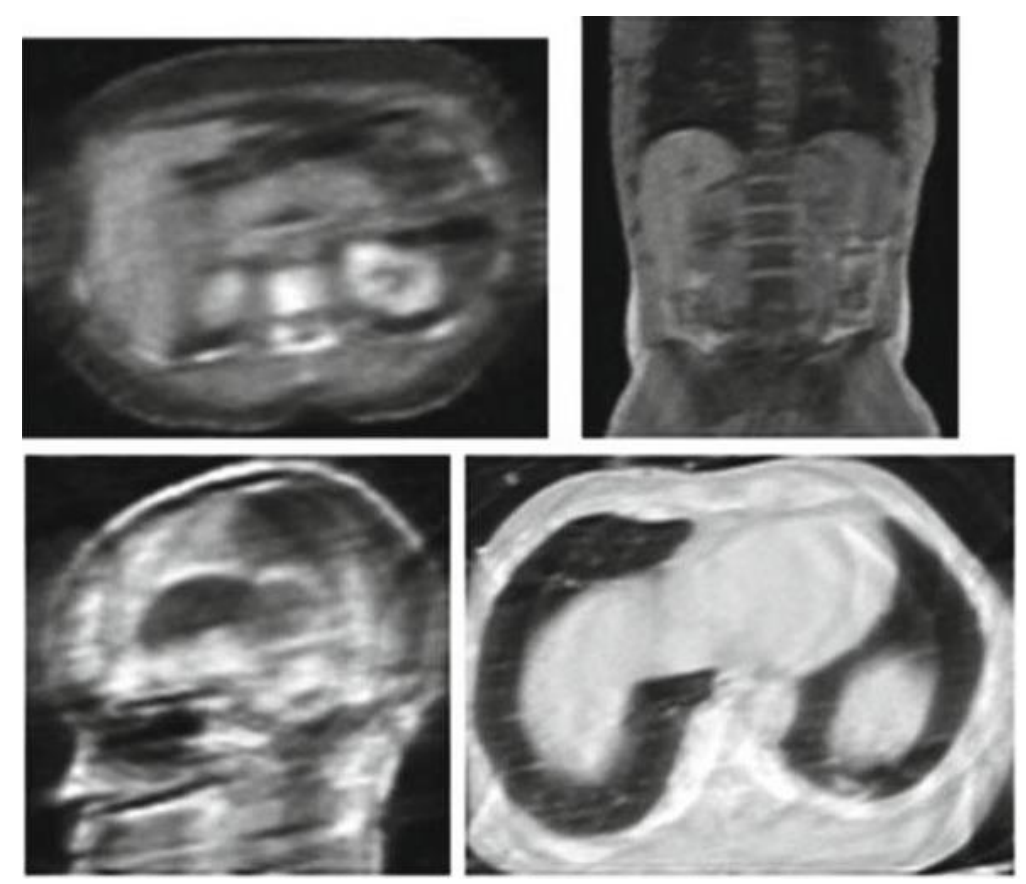

Figure 7: LRC images with PSF_two

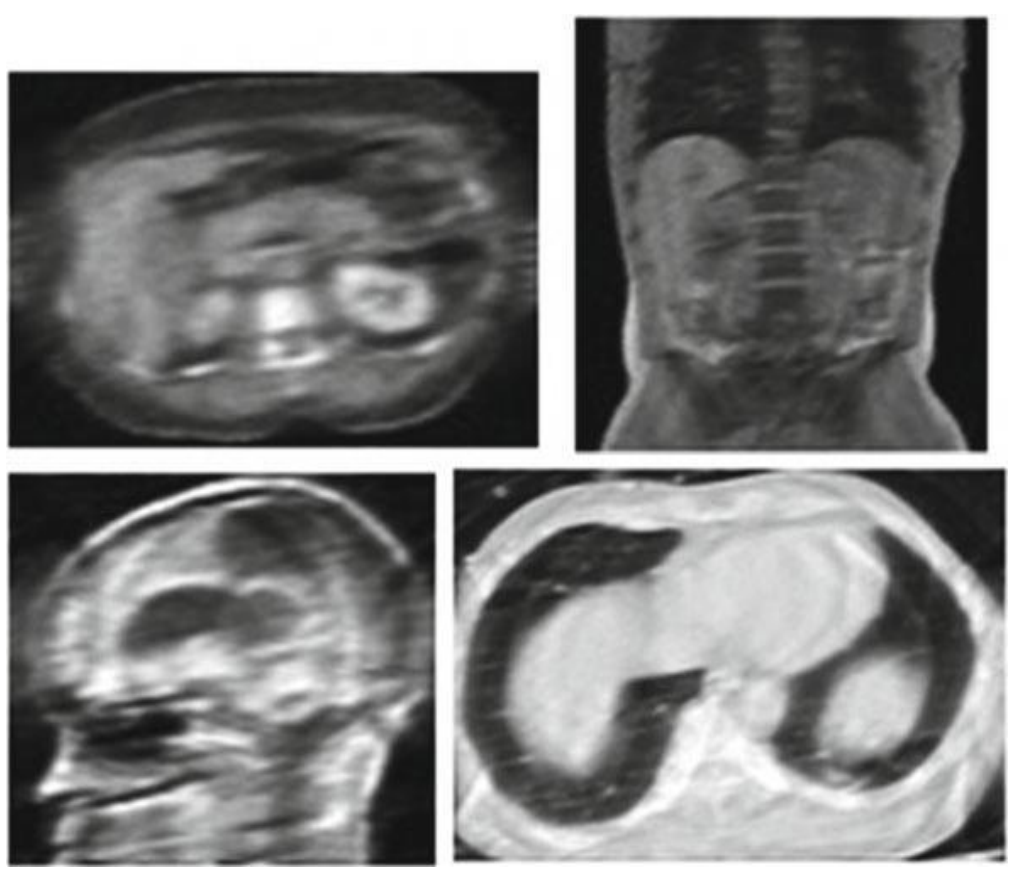

Figure 8: LRC images with PSF_three 
Eurasian Journal of Science \& Engineering EAJSE ISSN 2414-5629 (Print), ISSN 2414-5602 (Online)
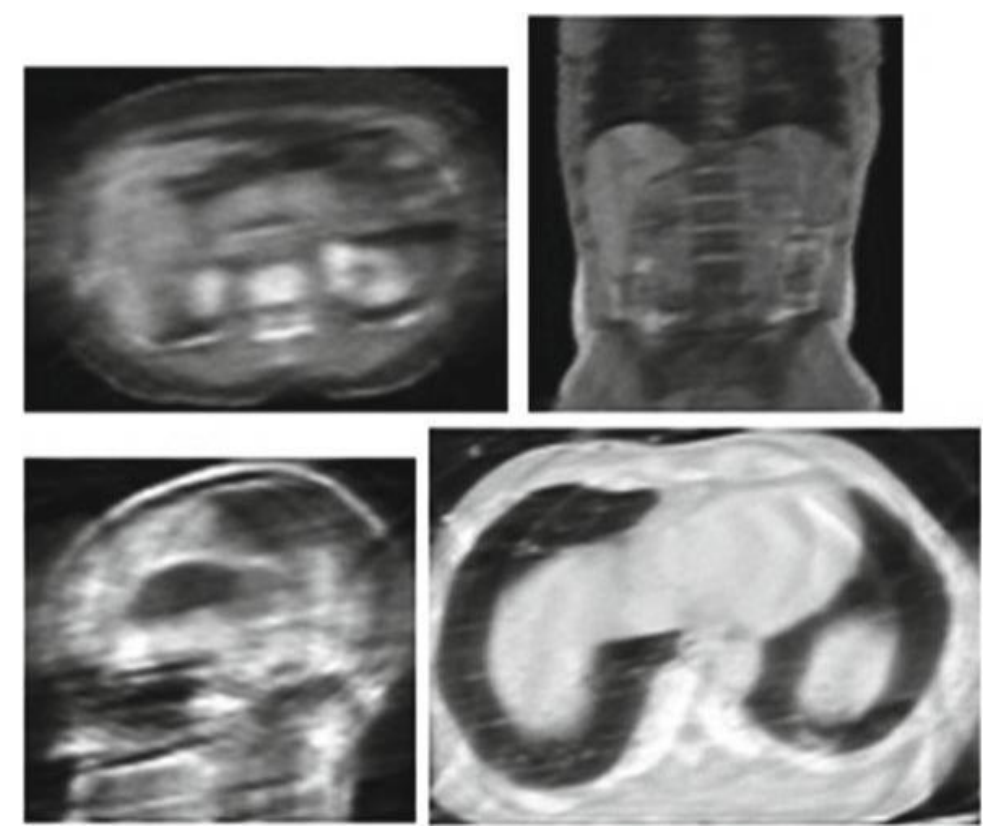

Figure 9: LRC images with PSF_four
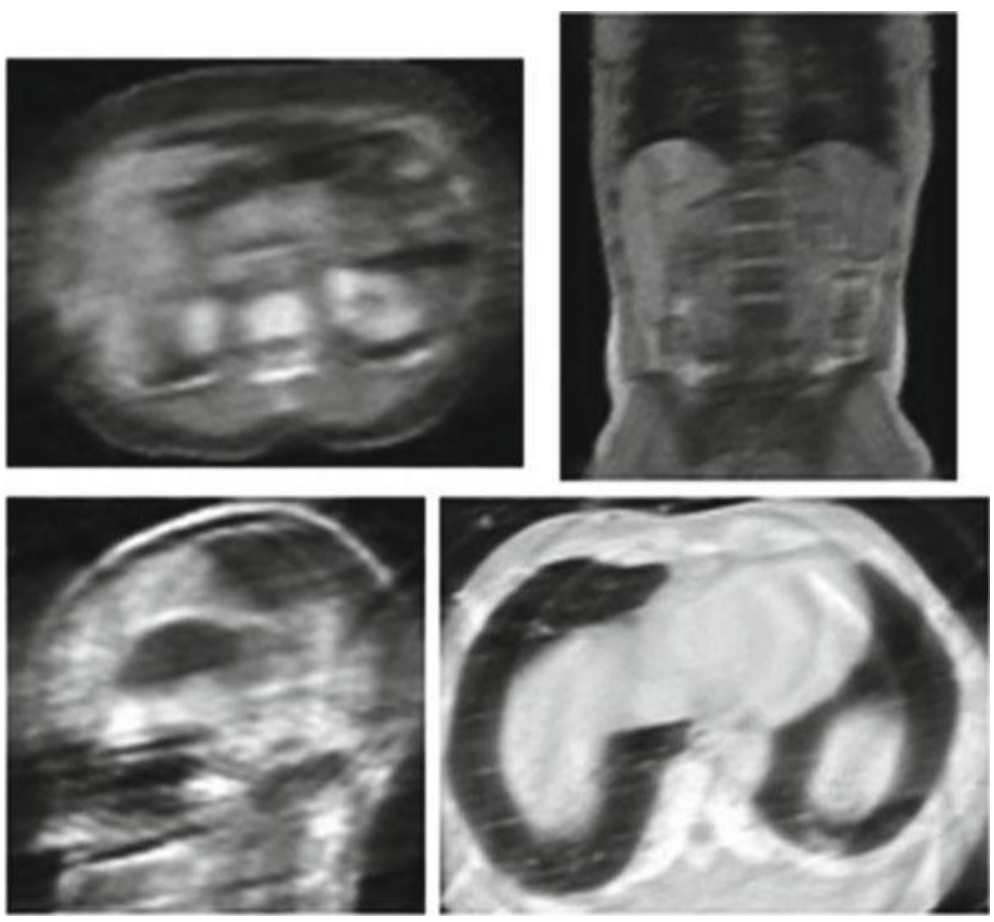

Figure 10: LRC images with PSF_five 

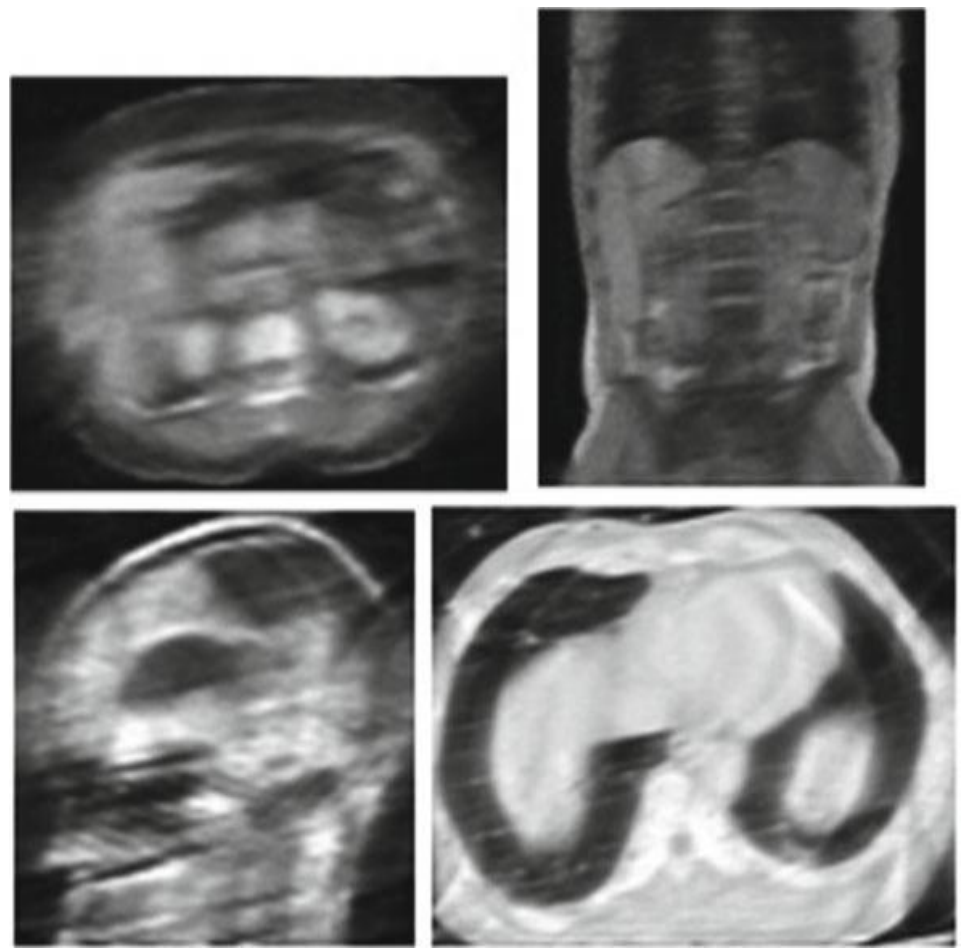

Figure 11: LRC images with PSF_six

\subsection{MSEC Methodology}

The mean mistake (Guo \& Wu, 2011) is determined with Dn characteristic qualities.

Error, ErN IPn-DnN. - Error

\section{MSEi_DnN MSEi(En). - MSquare}

The straight worth 12 to 17 signify $n, n$ is separate of PSF, en is a blunder for PSF, IPn is a crude picture or unique picture, and DnN is the complicated picture with PSF. The Instantaneous spatial mistakes are taken utilizing over the blunder condition. The square condition is used to figure the mistake of mean square. Each PSF, PSF_one to PSF_six, with the (EIDB) Efficient Image DeBlurringis is done to get un-obscured pictures, the MSEi on each picture known as MRIs and CTs are determined with worship to genuine pictures. Each instance of the mean square blunder esteems is given the accompanying tables 1 to 6 in the fourth characteristic of each table. The MSEi estimations with results appear in Tables 1 to 6 . Each table's outcomes are given markers for deviation from perfect Removing Complications. 
Table 1: MSE to PSF_one

\begin{tabular}{|l|l|l|l|}
\hline S. No. & Sample & After Blended & L PSF1 \\
\hline 1 & OG_MRI1 & 0.04075395 & 0.045567383 \\
\hline 2 & OG_MRI2 & 0.00328494 & 0.003998615 \\
\hline 3 & OG_MRI3 & 0.003829498 & 0.004788372 \\
\hline 4 & OG_CT1 & 0.008355153 & 0.009638349 \\
\hline 5 & OG_CT2 & 0.006004503 & 0.00711705 \\
\hline
\end{tabular}

Table 2: MSE to PSF_two

\begin{tabular}{|l|l|l|l|}
\hline S. No. & Sample & After Blended & L PSF1 \\
\hline 1 & OG_MRI1 & 0.040754 & 0.042521 \\
\hline 2 & OG_MRI2 & 0.003285 & 0.003321 \\
\hline 3 & OG_MRI3 & 0.003829 & 0.004023 \\
\hline 4 & OG_CT1 & 0.008355 & 0.010443 \\
\hline 5 & OG_CT2 & 0.006005 & 0.008825 \\
\hline
\end{tabular}

Table 3: MSE to PSF_three

\begin{tabular}{|l|l|l|l|}
\hline S. No. & Sample & After Blended & L PSF1 \\
\hline 1 & OG_MRI1 & 0.040754 & 0.041235 \\
\hline 2 & OG_MRI2 & 0.003285 & 0.004268 \\
\hline 3 & OG_MRI3 & 0.003829 & 0.005064 \\
\hline 4 & OG_CT1 & 0.008355 & 0.014442 \\
\hline 5 & OG_CT2 & 0.006005 & 0.007318 \\
\hline
\end{tabular}


Table 4: MSE to PSF_four

\begin{tabular}{|l|l|l|l|}
\hline S. No. & Sample & After Blended & L PSF1 \\
\hline 1 & OG_MRI1 & 0.040754 & 0.053368 \\
\hline 2 & OG_MRI2 & 0.003285 & 0.005824 \\
\hline 3 & OG_MRI3 & 0.003829 & 0.005644 \\
\hline 4 & OG_CT1 & 0.008355 & 0.019846 \\
\hline 5 & OG_CT2 & 0.006005 & 0.007309 \\
\hline
\end{tabular}

Table 5: MSE to PSF_five

\begin{tabular}{|l|l|l|l|}
\hline S. No. & Sample & After Blended & L PSF1 \\
\hline 1 & OG_MRI1 & 0.040754 & 0.041497 \\
\hline 2 & OG_MRI2 & 0.003285 & 0.004035 \\
\hline 3 & OG_MRI3 & 0.003829 & 0.004844 \\
\hline 4 & OG_CT1 & 0.008355 & 0.011953 \\
\hline 5 & OG_CT2 & 0.006005 & 0.007219 \\
\hline
\end{tabular}

Table 6: MSE to PSF_six

\begin{tabular}{|l|l|l|l|}
\hline S. No. & Sample & After Blended & L PSF1 \\
\hline 1 & OG_MRI1 & 0.040754 & 0.045643 \\
\hline 2 & OG_MRI2 & 0.003285 & 0.004074 \\
\hline 3 & OG_MRI3 & 0.003829 & 0.004845 \\
\hline 4 & OG_CT1 & 0.008355 & 0.009473 \\
\hline 5 & OG_CT2 & 0.006005 & 0.007119 \\
\hline
\end{tabular}

\subsection{Alpha Plane for Blending}

The compositing in the alpha plane (Wang \& $\mathrm{Ng}, 2012$ ) or picture mixing is obtained RGB weighted estimations of each pixel in a picture and adding a small amount of proportionate pixel of next picture RGB. On the off chance we have two therapeutic pictures as MRI1 and MRI2, every dot pixel, RGB values are determined for a composited picture of mixed (Pandey, 2017) as demonstrated as follows: 
Over all conditions, alpha factor (Igarashi, Yanagisawa, \& Togawa, 2015) Z (is $0-1$ territory esteems) is the profundity of mix for the blend picture. Similarly, the six restorative pictures are doing mixed together with neighbor equivalent alpha level to get a composited last complicated picture.

Each of the six PSF works over close to approach weightage, etc is MI2-MI4. MI1 is the last mixed picture. To assess the effectiveness of Removing Complication in addition to mixing plan, the MSEC for all mixed resultant restorative pictures (Bao, Fan, \& $\mathrm{Hu}, 2017$ ) for $\mathrm{CT}$ and MRI pictures tests are determined. The examination finished with L-RC complicated pictures as for mixed pictures. Table 7 shows examination and gives a base 10\% improvement in the recouped pictures on the off chance and they are mixed after Removing Complication.

Table 7: Comparison of MSEC worst case and blended

\begin{tabular}{|l|l|l|l|l|}
\hline S. No. & Sample & After Lucy & PSF_one & $\%$ Error Redu. \\
\hline 1 & OG_MRI1 & 0.04075 & 0.04556 & 10.3578 \\
\hline 2 & OG_MRI2 & 0.00328 & 0.00399 & 14.568 \\
\hline 3 & OG_MRI3 & 0.00382 & 0.00478 & 16.8558 \\
\hline 4 & OG_CT1 & 0.00835 & 0.00963 & 12.1755 \\
\hline 5 & OG_CT2 & 0.00069 & 0.00711 & 13.8764 \\
\hline
\end{tabular}

\section{Experimental Result}

The missing count is done, and it gives a decent estimation. The recouped pictures of noteworthy improvement results appear in Table 7. This work has been actualized in a reproduction situation utilizing MATLAB device. The MSE equation is utilized for mistake examination. Figure 12 shows the resultant de-obscured CT and MRI pictures obtained subsequent to mixing activity which is applied to L-RC complicated restorative pictures.

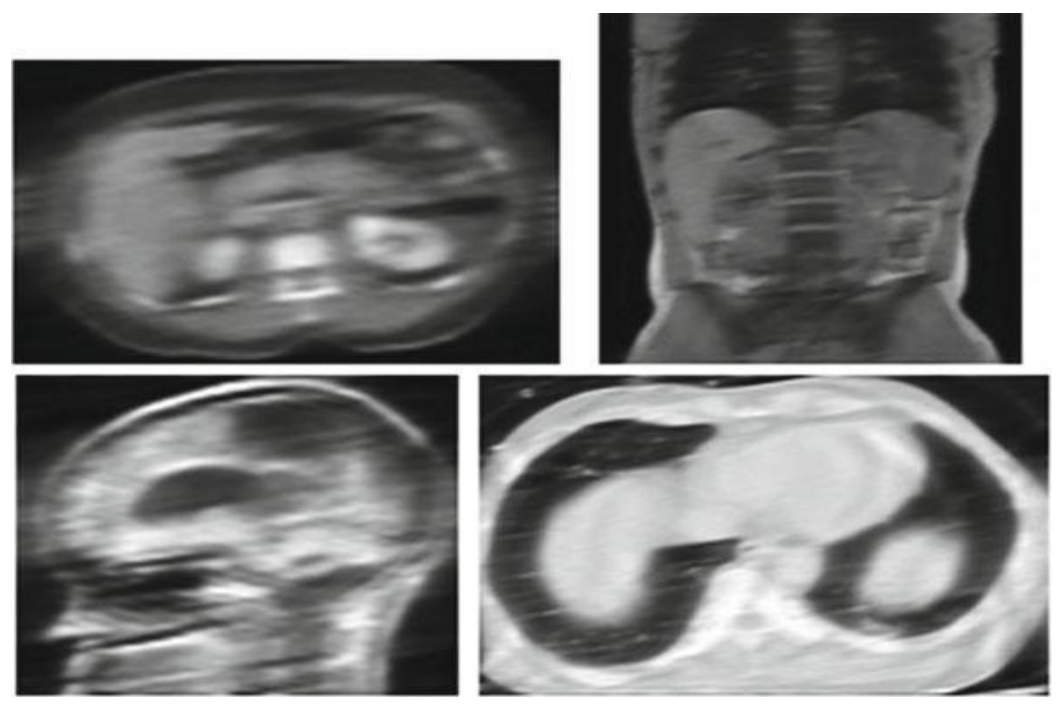

Figure 12: Final Image of De-blurred 
Here we investigated the estimation error using the proposed method, respectively, under different noise levels. Figure 13 and 14 illustrate the final identification result of the blur, which indicates that the proposed identification method can achieve satisfactory results.

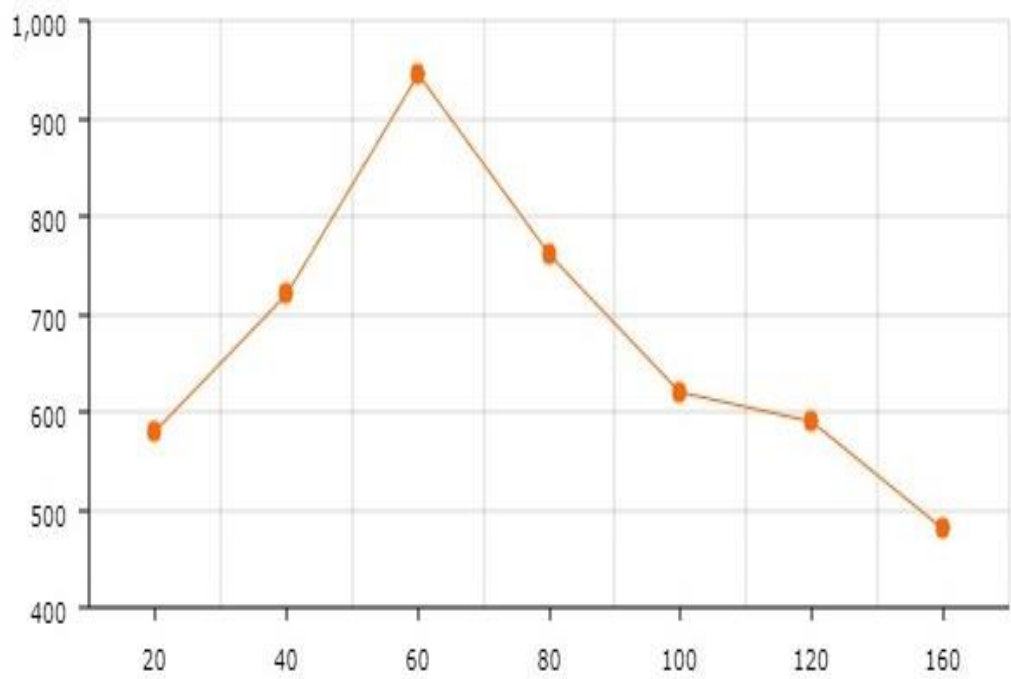

Figure 13: Estimation result of motion angle

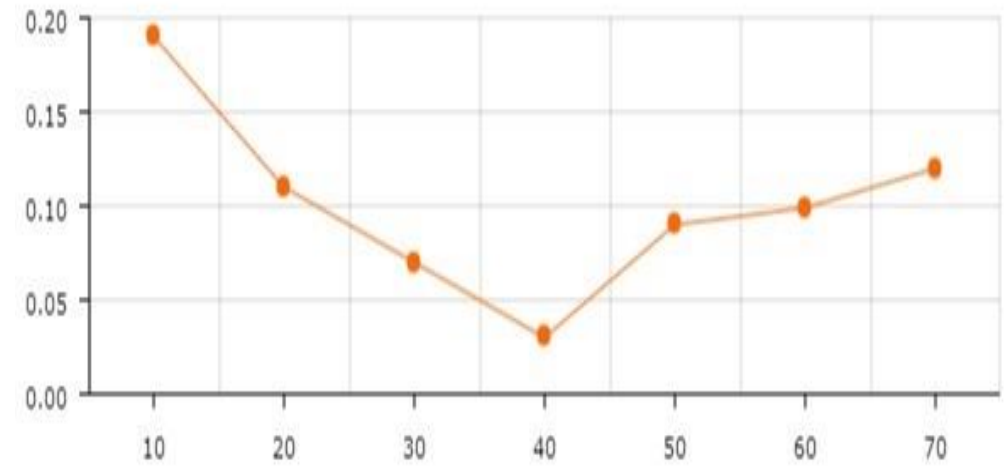

Figure 14: Estimation Result of motion length

According to these experimental results, our proposed method in the presence of complex noises performs well, which indicates the robustness and efficiency of our algorithm.

\section{Conclusion}

This proposed approach has been demonstrated using EIDB and point spread function deblurring concepts. De-obscuring of the MRI and CT images, which were hitherto obscured, has been achieved. The alpha plane mixing has done the extent of critical enhancement for de-obscured therapeutic medical imaging. The mixing factor in compositing the medicinal pictures has been utilized as around equivalent weightages. The de-obscuring of CT and MRI restorative images is justifying the fact that the proposed approach is capable of reducing image blurring even in complicated medicinal imaging. In this manner, the work adds to the advancement in restorative imaging through this proposed work using differed PSF Removing Complication with alpha plane blending (ABP). 


\section{References}

Angelis, G., J. G., \& Kyme, A. Z. (2016). Modelling the motion dependent point spread function in motion corrected small animal PET imaging. Nuclear Science Symposium, Medical Imaging Conference and Room-Temperature Semiconductor Detector Workshop.

Bao, J., Fan, J., \& Hu, X. (2017). An effective consistency correction and blending method for camera-array-based microscopy imaging. WSSIP International Conference on Systems, Signals and Image Processing.

Dong, B., \& Xie, S. (2016). A method of point spread function estimation for wideband electromagnetic distribution. Pacific International Symposium Electromagnetic Compatibility (APEMC).

Fowler, B., ElGamal., A.,\& Yang, D. (1994). A CMOS area image sensor with pixel-level A/D Conversion. IEEE International Conference on ISSCC. IEEE.

Guo, D., \& Wu, S. Y. (2011). Estimation in Gaussian noise: properties of the minimum mean-square error. IEEE Trans. Inform. Theory.

Igarashi, K., Yanagisawa, M., \& Togawa, N. (2015). Image synthesis circuit design using selectorlogic-based alpha blending and its FPGA implementation. 11th IEEE International Conference ASIC (ASICON) .

Jiunn-Lin, W., Chang, C. F., \& Chen, C. S. (2013). An adaptive Richardson-Lucy algorithm for single image Deblurring using local extrema filtering. J. Appl. Sci. Eng. 269-276.

Li, B., \& Zhan, Z. (2012). Research on motion blurred image restoration. 5th International Congress on Image and Signal Processing (CISP). IEEE.

Lil, W., Meunier, J., \& Soucy, J. P. (2005). A 3D adaptive Wiener filter for restoration of SPECT images using MRI as reference images. 27th IEEE Conference in Medicine and Biology. Shanghai, China: IEEE.

Mane, A. S., \& Pawar, M. M. (2014). Removing blurring from degraded image using blind Removing Complication with canny edge detection technique. Int. J. Innov. Res. Adv. Eng. (IJIRAE), 2349-2163.

Pandey, A. P. (2017). Development of saliency-based seamless image compositing using hybrid blending (SSICHB). IET Image Process.

Pizurica, A., \& Philips, W. (2003). A versatile wavelet domain noise filtration technique for medical imaging. IEEE Trans. Med. Imaging.

Schretter, C., Bundervoet, S., \& Blinder, D. (2016). Ultrasound imaging from sparse RF samples using system point spread functions. IEEE Trans Ultrason. Ferroelectr. Freq. Control. Retrieved from https://doi. org/10.1109/tuffc.2017.2772916

Sharma, P. S. (2016). An MSE (mean square error) based analysis of deconvolution techniques used for deblurring / restoration of MRI and CT Images. ICPS Proceedings .

Wang, W., \& Ng, M. (2012). A variational method for multiple-image blending. IEEE Trans. Image Process.

Yim, P., Choyke, P., \& summers, R. (2000). Gray-scale skeletonization of small vessels in mag-netic resonance angiography. IEEE Trans. Med. Imaging. 